\title{
Editorial: Topical Volume on Gamma Ray Bursts-A Tool to Explore the Young Universe
}

\author{
Diego Götz ${ }^{1}$ Maurizio Falanga ${ }^{2}$
}

Published online: 22 November 2016

(C) Springer Science+Business Media Dordrecht 2016

This topical volume reviews a comprehensive set of problems raised by the use of GammaRay Bursts (GRBs) as cosmological probes, as they were addressed and debated during the ISSI-Beijing workshop "Gamma Ray Bursts: a tool to explore the young Universe", April 13-17, 2015 in Beijing. Particularly original about this workshop was that the invited scientists were representing both the GRB community stricto sensu, as well as the community studying high-redshift galaxies and star formation history.

Despite the recent progresses in Gamma-Ray Burst science, obtained in particular thanks to the Swift and Fermi satellites, there are still many open questions in the field. One concerns the mechanisms that power these extreme explosions (in a handful of seconds the isotropic equivalent energy emitted by GRBs spans from $10^{50}$ to $10^{54} \mathrm{erg}$, making them the most luminous events in the Universe), which is still unclear after more than four decades since their discovery. In particular the content of the relativistic flow that produces the GRBs remains to be investigated: especially in terms of its bulk Lorentz factor, its magnetization, its baryon loading and their consequences on the possibility of GRBs being the sources of Ultra High Energy Cosmic Rays (UHECRs).

Another open issue concerns the nature of GRBs progenitors: while thanks to a handful of spectroscopic associations with type I c Supernovae, long GRBs are currently considered the endpoint of very massive ( $>30-50$ solar masses) stars, the situation is less clear for what concerns the short GRBs. The most popular models involve the possibility of a coalescence of two compact objects (black holes or neutron stars), but a direct proof of this model is still lacking.

In addition to particle acceleration, radiation physics, and stellar evolution, GRBs are also pertinent to other branches of astrophysics, like cosmology. Indeed, thanks to their bright afterglows they allow the observers to pinpoint the most distant galaxies, and to study them thanks to the imprinting on the afterglow data of the GRB close environment and the

D. Götz

diego.gotz@cea.fr

1 CEA Saclay_DRF/Irfu/Service d'Astrophysique, 91191 Gif-sur-Yvette, France

2 ISSI-Beijing, Beijing, China 
intervening matter between the GRB and the Earth, gathering a wealth of information on the structure and physical state of the gas in the Universe through absorption lines.

This aim of the ISSI-Beijing workshop was to elucidate the connection between GRBs and the deep Universe. In recent years much effort has been put into the ground based followup of GRB afterglows: the median measured redshift of long GRBs is $\sim 2$ (with the highest value reported to date of $\sim 9.4$ ), which means that GRBs are among the best candidates to study the deep Universe. Ten years after the launch of the NASA Swift satellite, statistics on GRBs above $z=3$ have been constantly improving, reaching more than 40 events to date. It was hence timely to coherently review and assess the current knowledge on GammaRay Bursts, their progenitors, and their environment, in order to understand their nature and origin. Understanding the GRB phenomenon globally is of paramount importance for the use of GRBs as beacons of the deep Universe.

At the same time, we felt it would be wise to start thinking about how to develop worldwide synergies between the high-redshift GRB studies and the surveys of the deep Universe, especially in the framework of the future GRB dedicated facilities in which China is deeply involved. This workshop was also labeled as scientific event of the celebration of the 50th anniversary of the diplomatic relationship between France and China, and is related to the more general framework of the upcoming Sino-French space mission SVOM (Space based Variable astronomical Object Monitor) dedicated to GRBs and time domain astronomy studies.

The paper by Zhang et al. contains valuable introductory material about the GRBs observational properties and the associated theoretical models, and provides the basis for the following sections. The review by Levan et al. deals with the GRB progenitors, covering the different theories about what parent star(s) could be at the origin of these peculiar objects. The paper by Nagamine et al. summarizes our current knowledge on galaxy formation and stellar evolution especially at early times. Perley et al. review the characteristics of the galaxies hosting GRBs. The paper by Cucchiara et al. provides some examples about the use of GRBs as cosmological probes, using them as powerful background lighthouses shining through the Universe. Toma et al. investigate the possibility of producing and detecting GRBs from the first (so called population III) stars. The paper by Chary et al. deals with the possible use of GRBs as (unbiased?) tracers of the early star formation. Wang et al. review the progress on the possibility of probing fundamental cosmology and physics using GRBs. Finally, Yuan et al. provide an interesting perspective on the future facilities that could tackle the observation of highly redshifted GRBs such as SVOM, THESEUS, Einstein Probe, SKA, WFIRST, JWST, ELTS, etc.

It is our honor to warmly compliment the conveners and organizers of the workshop; they conducted their job of producing this extensive review volume with great enthusiasm and dedication. The fast growing space research community in China and elsewhere in Asia is eager to increase its scientific exchanges with the colleagues from other parts of the world, and, at the same time, scientists from outside Asia will find new opportunities in Asia/China through ISSI-Beijing. So, 46 highly respected scientists from all over the world representing different scientific communities could be gathered to work on these review papers. We thank all who participated and who contributed to the papers. This excellent set of review papers represents an important outcome of ISSI-Beijing: congratulation to all. 